Annales Geophysicae (2002) 20: 321-327 (c) European Geophysical Society 2002

\title{
Interball-tail observations of vertical plasma motions in the magnetotail
}

\author{
A. A. Petrukovich ${ }^{1}$ and Y. I. Yermolaev ${ }^{1}$ \\ ${ }^{1}$ Space Research Institute, 84/32 Profsoyuznaya St., Moscow, 117810, Russia
}

Received: 23 January 2001 - Revised: 16 May 2001 - Accepted: 29 May 2001

\begin{abstract}
The Interball spacecraft configuration favors, in contrast to previous experiments, investigation of vertical ion flows $\left(\mathrm{GSM} V_{z}\right.$ ). We use measurements of the CORALL instrument for the statistical study of $V_{z}$ and $V_{y}$ plasma flows in the mid-tail plasma sheet. In agreement with the previous observations, the mean $V_{y}$ was positive on the dusk side and negative on the dawn side. When IMF was southward, the mean $V_{z}$ consisted of the convection flow towards the equatorial plane $\sim 7 \mathrm{~km} / \mathrm{s}$ and the northward flow $\sim 8 \mathrm{~km} / \mathrm{s}$. When IMF was northward, both components nearly vanished. The velocity variance was much larger than the mean values. The $V_{z}$ variance maximized on the dawn flank and was always 15-20\% smaller than the $V_{y}$ one. The $V_{y}$ variance maximized in the pre-midnight sector closer to the neutral sheet. We conclude that velocity fluctuations are composed with the inherent high-beta plasma turbulence contributing to all components, and the BBF-related activity contributing mainly to $V_{y}$ in the pre-midnight plasma sheet.
\end{abstract}

Key words. Magnetospheric physics (magnetotail; plasma sheet; plasma convection)

\section{Introduction}

The plasma dynamics in the Earth's magnetotail remains the focus of research in space plasma physics for many years. Under southward IMF, the solar wind flow drags the magnetic field lines (reconnected on the dayside) around the magnetosphere in the anti-sunward direction, and the pattern is closing in the magnetotail with the vertical equatorward and then a horizontal earthward convection (Dungey, 1961). The first direct experimental evidence of such convection was provided with the AMPTE/IRM and ISEE-2 observations (Angelopoulos et al., 1993) of mean ion flows in the $X Y$ plane, which were of the order of $\sim 10-50 \mathrm{~km} / \mathrm{s}$ (hereafter GSM frame of reference is used).

Correspondence to: A. A. Petrukovich

(apetruko@iki.rssi.ru)
Such a quasi-steady flow pattern appears in the experimental data only after averaging over large spatial and temporal intervals. On smaller time scales, it is mixed with local variations. Discovery of the fast earthward plasma flows (Baumjohann et al., 1990; Angelopoulos et al., 1992), termed as bursty bulk flows (BBFs), was an important step in our understanding of the magnetotail as of a highly variable system. Based on the AMPTE/IRM and ISEE-2 data, Angelopoulos et al. (1994) defined BBFs as segments of continuous ion flow velocity above $100 \mathrm{~km} / \mathrm{s}$, with maximal velocity above $400 \mathrm{~km} / \mathrm{s}$. The dominant velocity component was $V_{x}$, but $V_{y}$ was also often substantial. BBFs lasted for about $7-15 \%$ of the observation time with $\sim 550 \mathrm{~s}$ of median duration. Sergeev et al. (1996) associated BBFs with the small-scale reconnection activity, while Pontius and Wolf (1990) associated BBFs with the interchange instability.

On a smaller time scale of the order of $1 \mathrm{~min}$, Sergeev and Lennartsson (1988) and Angelopoulos et al. (1993) discovered that ion flows are very variable both inside and outside BBFs, so that the variance of these seemingly random fluctuations is much larger than the average velocity outside BBFs. Borovsky et al. (1997) analyzed ISEE-2 measurements of ion flows and the magnetic field and concluded that $V_{x}$ and $V_{y}$ flows are strongly turbulent and typically sub-Alfvénic. The occurrence distribution of flow velocities was non-Gaussian due to the presence of a large number of high-velocity samples. Angelopoulos et al. (1999) used the Geotail data set to suggest that earthward velocity bursts (BBFs) drive the rest of the plasma sheet fluctuations, so that the self-organized critical state is formed.

So far, due to the instrument's limitations, statistical analysis of the flow velocity measurements was performed only for the $X$ and $Y$ directions. However, $V_{z}$ velocity data should contain signatures of the convection towards the equatorial plane, while a comparison of fluctuations along all three directions might help to understand the nature of the plasma sheet turbulence (Yermolaev et al., 2000). The Interball-Tail spacecraft provided the unique possibility of reliable measurements of $V_{z}$ (and $V_{y}$ ) ion flow components. In this paper, 

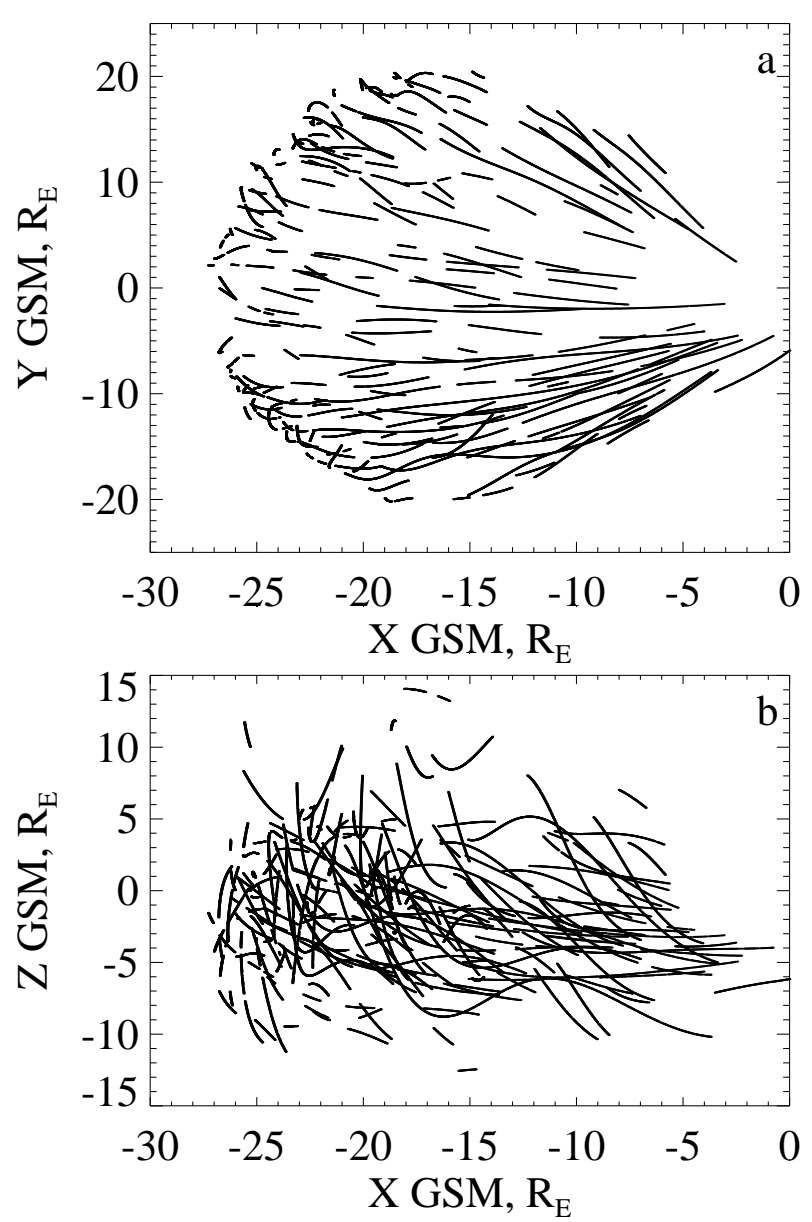

Fig. 1. Positions of the Interball-Tail plasma sheet measurements: $X Y$ (a) and $X Z$ (b) GSM planes.

we analyze the plasma sheet ion flow data collected during four years of the Interball-Tail operation.

\section{Instrumentation and data selection}

The ion spectrometer CORALL (Yermolaev et al., 1997) on board the Interball-Tail (IT) spacecraft detected ion flux in the energy range $0.05-25 \mathrm{keV}$ with the use of the $2 \mathrm{D}$ fivesector electrostatic analyzer. The whole 3D ion distribution function was scanned during the spacecraft spin. The IT spin period was about $2 \mathrm{~min}$ and the spin axis was pointed approximately sunward.

With such a spacecraft configuration, all directions in the $Y Z$ GSM plane were covered by the same sensors, and measured $V_{y}$ and $V_{z}$ ion flow velocities were free from artificial offsets. Thus, investigations of vertical plasma flows were favored on board IT, as compared with the ion spectrometers on board ISEE-2 and AMPTE/IRM (Bame et al., 1978; Paschmann et al., 1985). It should be noted that the ion flux was not measured inside two $\pm 16^{\circ}$-wide cones centered at the spin axis. During plasma moment calculations, the spe-
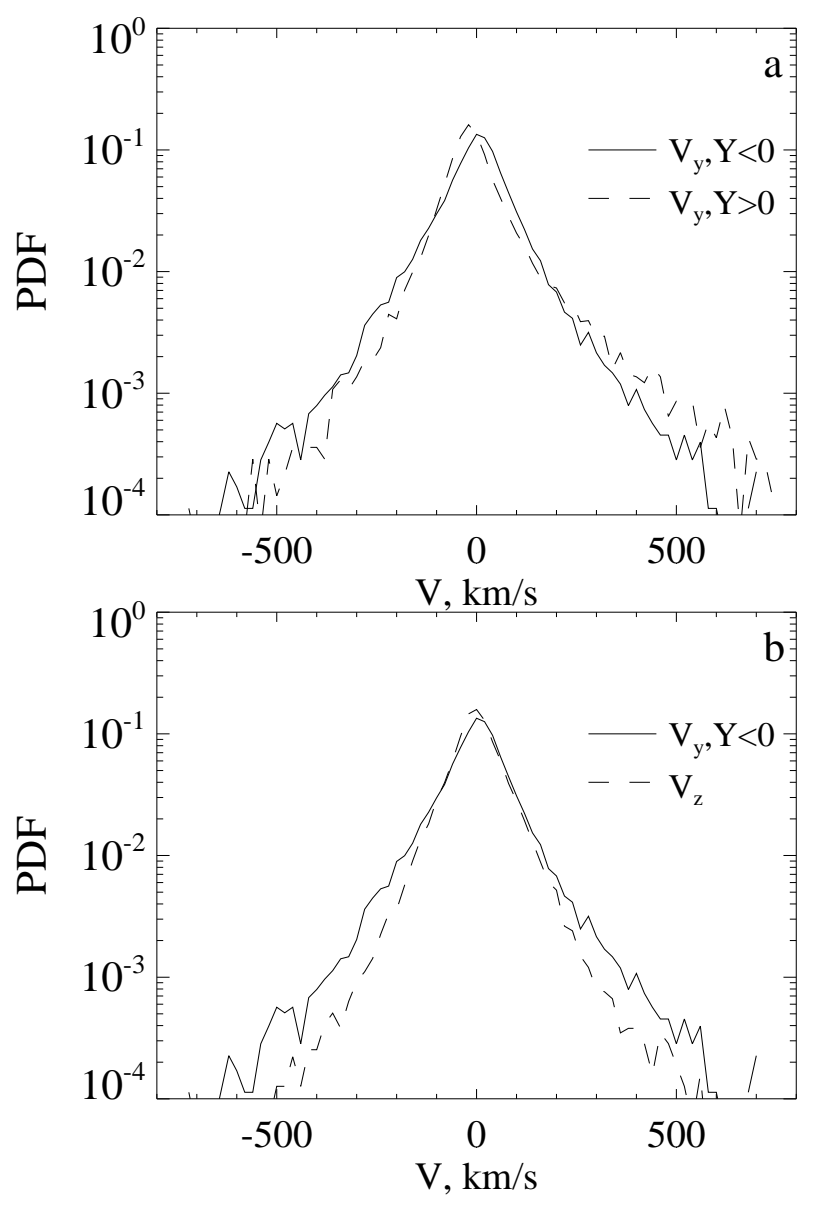

Fig. 2. Comparison of PDFs: (a) $V_{y}$ for $Y>0$ and $V_{y}$ for $Y<0$; (b) $V_{z}$ and $V_{y}$ for $Y<0$.

cial algorithm was implemented to account for the flux in these blind zones, but, nevertheless, values of ion density and $V_{x}$ velocity were not so accurate. Therefore, we concentrated in our analysis on measurements of $V_{y}$ and $V_{z}$ components.

According to the annual orbit evolution, IT visited the magnetospheric tail during the fall-winter seasons of the Northern Hemisphere (October-January). We included in the plasma sheet data set all data samples that (1) had count rates above the background, (2) were not identified as a part of the plasma sheet boundary layer, LLBL, magnetosheath or mantle, (3) belonged to continuous plasma sheet intervals longer than $1 \mathrm{~h}$. As the instrument did not observe anti-sunward streaming cold particles, it returned zero counts (within the background noise level) in the magnetotail lobe.

Each two-minute sample of plasma moments (moments were computed on the ground) was combined with the corresponding local magnetic field and spacecraft position vectors. The magnetic field on board IT was measured by the ASPI/MIF-M instrument (Klimov et al., 1997), and in this study we used the 2-min averaged data. IMF and solar wind (SWE) data measured by Wind spacecraft were taken from 
Table 1. Moments of $V_{y}, V_{z}$ PDFs. Skewness and kurtosis are normalized by the variance. $90 \%$-confidence intervals are included for mean and variance

\begin{tabular}{lccc}
\hline Subset & $V_{y}, Y>0$ & $V_{y}, Y<0$ & $V_{z}$ \\
\hline Number of points & 7006 & 10232 & 17238 \\
Mean, km/s & $10.0 \pm 1.5$ & $-13.3 \pm 1.5$ & $8.1 \pm 0.9$ \\
Variance, $\mathrm{km} / \mathrm{s}$ & $76.0 \pm 2.1$ & $90.7 \pm 2.1$ & $68.3 \pm 1.2$ \\
Skewness & 1.51 & -0.16 & 0.2 \\
Kurtosis & 15.7 & 9.78 & 9.6 \\
\hline
\end{tabular}

the CDAWeb public database and shifted by convection time necessary for the solar wind to cover the distance between Wind and IT.

The collected set of the plasma sheet measurements during 1995-1998 totaled about 32000 points. The spacecraft positions ranged from 0 to $-27 R_{E}$ in $X$ coordinate, from -21 to $+21 R_{E}$ in $Y$, and from -15 to $15 R_{E}$ in $Z$ (Fig. 1). Due to the solar minimum period, corresponding geomagnetic conditions were generally quiet or slightly disturbed ( $K_{p}$ values within $4+$ ). IMF orientation was almost evenly distributed between northward and southward directions.

It is known from previous investigations (Borovsky et al., 1997) that velocity variance is of the order of $100 \mathrm{~km} / \mathrm{s}$, while the expected large-scale convection velocity is $\sim 10 \mathrm{~km} / \mathrm{s}$. For the number of data samples $N \gg 1$, the $90 \%$ confidence range for the mean velocity estimate (of a Gaussian process) is $\delta V \approx 1.6 \cdot \sigma / \sqrt{N}$, where $\sigma$ is the estimate of variance. Therefore, to keep $\delta V$ within a few $\mathrm{km} / \mathrm{s}$, one has to include at least several thousands of data samples in the calculation. Due to such a requirement, the smallest reasonable size of a subset selected, for example, to study spatial dependence of flow velocities, was actually equal to one-eighth of the full set.

In the rarefied plasma, detected flow fluctuations might include both ambient plasma flow variations and statistical variations of the count rate caused by insufficient sensitivity of an instrument. In this investigation we directly excluded low count rate data samples. It is noteworthy in retrospect, that introduction of such a threshold appeared to affect only the values of the variance of velocity fluctuations, while the values of mean velocities and the ratios of variances for different components remained almost unchanged.

\section{Observations}

\subsection{Probability distributions and variance}

The probability distribution function (PDF) of $V_{y}$ and $V_{z}$ measurements are presented in Fig. 2. All PDFs have nonGaussian shapes, but the wings of the $V_{z}$ distribution are lower than that of the $V_{y}$ ones. The main difference between the $V_{y}$ PDFs for $Y>0$ (dusk) and $Y<0$ (dawn) is the larger probability to observe duskward high-speed flows at
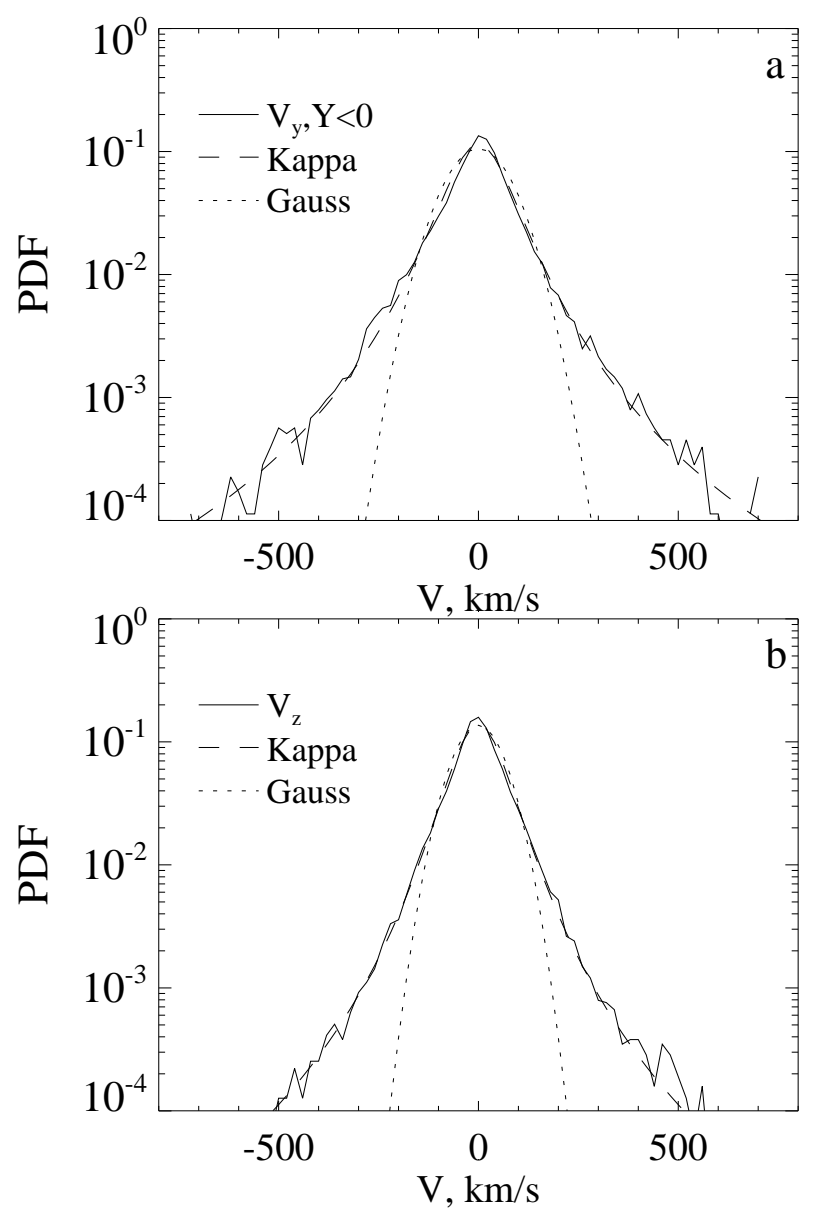

Fig. 3. Comparison of the experimental PDFs with the Gaussian and kappa function fits: (a) $V_{y}$ for $Y<0$; (b) $V_{z}$.

$Y>0$. There were no significant differences between the $V_{z}$ PDFs for dusk and dawn. Distributions are described in Table 1 by their moments. The mean velocities are of the order of $10 \mathrm{~km} / \mathrm{s}$ and the variances are of the order of $50-100 \mathrm{~km} / \mathrm{s}$. These two characteristics will be discussed in more detail below. The third and fourth moments, skewness and kurtosis, are normalized by the value of the variance and illustrate a deviation of a distribution from the Gaussian shape. Detected large values of kurtosis $\sim 10$ reflect the presence of the significant high-velocity wings. (Kurtosis of a Gaussian PDF is equal to unity.) The finite skewness ( 0 for a Gaussian case) describes the non-symmetry that remains after subtraction of the mean velocity. Both dawn-side $V_{y}$ and $V_{z}$ PDFs are almost symmetric with the small skewness, while the presence of high-speed duskward directed flows contributes to the significant skewness value for the dusk-side $V_{y}$ PDF.

Our experimental PDFs are readily fitted by the kappa distribution $f(v)$ in Fig. 3 (e.g. Vasyliunas, 1968):

$$
f(v) \sim\left(1+v^{2} /\left(k \cdot V_{c}^{2}\right)\right)^{-(k+1)} .
$$

The Gaussian distribution is the particular case of kappa dis- 
Table 2. Results of the kappa distribution fit

\begin{tabular}{lccc}
\hline Subset & $V_{y}, Y>0$ & $V_{y}, Y<0$ & $V_{z}$ \\
\hline$k$ & 0.86 & 1.26 & 1.72 \\
$V_{c}, \mathrm{~km} / \mathrm{s}$ & 69.3 & 98.5 & 80.6 \\
Error & 3.71 & 1.97 & 1.31 \\
\hline
\end{tabular}

tribution with $k=\infty$. In Table $2, k$ and $V_{c}$ parameters of the kappa function (determined during the least squares fit) are given. An almost twofold increase of the error for the duskside $V_{y}$ is explained by the non-symmetry of the considered distribution. Figure 3 also includes Gaussian fits, which constitute very poor representation of the data.

In Fig. 4, we present the variances of velocity fluctuations computed in several spatial bins. The whole range of available $Y$ spacecraft positions was divided into the four subranges $Y<-15 R_{E}$ (dawn flank), $-15 R_{E} E<Y<0$ (dawn center), $0<Y<15 R_{E}$ (dusk center), $Y>15 R_{E}$ (dusk flank). In addition, we separated the inner plasma sheet (the part close to the magnetic field reversal) and the outer plasma sheet regions. In previous investigations (Angelopoulos et al., 1993) the threshold $\beta=1$ or $\beta=0.5$ ( $\beta$ is the ratio of ion thermal to magnetic energy) was used to select inner and outer plasma sheet regions. Since ion density was not always measured reliably in the CORALL experiment, we substituted this $\beta$ criterion with the similar magnetic field criterion. All data with the local magnetic field magnitude $|B|<10$ nT were attributed to the inner plasma sheet region. Plasma with density $0.1 \mathrm{~cm}^{-3}$, ion temperature $2000 \mathrm{eV}$, and magnetic field $10 \mathrm{nT}$, has ion $\beta \sim 0.8$.

The $V_{z}$ variance values were always smaller than the $V_{y}$ ones. There was no significant difference between the $V_{z}$ subsets in the outer and inner plasma sheet, but the $V_{z}$ variance on the dawn side $(\sim 80 \mathrm{~km} / \mathrm{s})$ was typically larger than that on the dusk side $(\sim 60 \mathrm{~km} / \mathrm{s})$. On the contrary, the $V_{y}$ fluctuations in the inner plasma sheet were $\sim 20 \%$ higher than that in the outer plasma sheet and had the pronounced maximum in the pre-midnight sector $(\sim 115 \mathrm{~km} / \mathrm{s})$. There was no significant dependence of variance on other parameters, such as local magnetic field $B_{z}$, IMF, or $X$ coordinate.

\subsection{Mean velocities}

First of all, we derive the mean $V_{y}$ velocities $\left(\left\langle V_{y}\right\rangle\right)$ in the same spatial bins as during the variance analysis (Fig. 5). The values of $\left\langle V_{y}\right\rangle$ significantly vary with the spacecraft position. Everywhere on the dawn side, $\left\langle V_{y}\right\rangle \sim-15 \mathrm{~km} / \mathrm{s}$ (average flow is directed dawnward). On the dusk side, $\left\langle V_{y}\right\rangle$ $\sim 10 \mathrm{~km} / \mathrm{s}$ (average flow is directed duskward), except the pre-midnight sector of the inner plasma sheet, where $\left\langle V_{y}\right\rangle$ $\sim 25 \mathrm{~km} / \mathrm{s}$. This result is primarily important to ensure compatibility of our analysis with the previous investigations.

The $\left\langle V_{z}\right\rangle$ values in the plasma sheet were of the order of $5-10 \mathrm{~km} / \mathrm{s}$ (Fig. 5). Since the directions of the expected equa-

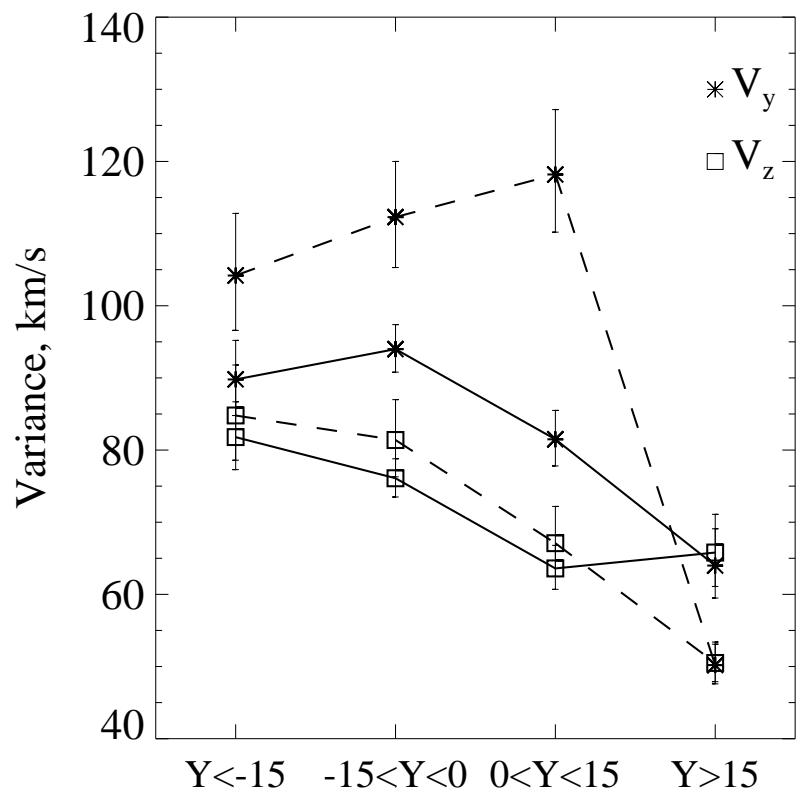

Fig. 4. Variances of $V_{y}$ (stars) and $V_{z}$ (squares) flows in the inner (dashed curves) and the outer (solid curves) plasma sheet with respect to the $Y$ GSM spacecraft position.

torward (related to the global magnetospheric convection) drift $V_{e}$ are opposite in the northern and southern parts of the magnetotail, mean velocities were calculated separately in each part $\left(\left\langle V_{z}^{+}\right\rangle\right.$and $\left\langle V_{z}^{-}\right\rangle$, respectively). We used the sign of the local magnetic field component $B_{x}$ as the criterion of the spacecraft position with respect to the current sheet. We also limited our analysis to the region $X<-10 R_{E},-15 R_{E}$ $<Y<15 R_{E}$. Then,

$\left\langle V_{z}^{+}\right\rangle=V_{d}-V_{e},\left\langle V_{z}^{-}\right\rangle=V_{d}+V_{e}$,

and

$V_{e}=0.5 \cdot\left(\left\langle V_{z}^{+}\right\rangle-\left\langle V_{z}^{-}\right\rangle\right), V_{d}=0.5 \cdot\left(\left\langle V_{z}^{+}\right\rangle+\left\langle V_{z}^{-}\right\rangle\right)$.

Here, $V_{d}$ is the feasible flow component, which does not depend on the $Z$ position of the spacecraft. $\left\langle V_{z}^{+}\right\rangle,\left\langle V_{z}^{-}\right\rangle, V_{d}, V_{e}$ values were computed for the northward and southward IMF cases separately (Table 3). We used instant values of IMF to form these subsets. The attempt to use IMF values, averaged over 1-h intervals preceding every sample, did not result in any significant changes. In the fifth column of Table 3 , the $90 \%$ confidence range of the mean velocity is included $(\delta V)$. Both $V_{d}$ and $V_{e}$ were found to be close to zero (with the $90 \%$ confidence range) for the northward IMF and were equal to $\sim 7-9 \mathrm{~km} / \mathrm{s}$ for the southward IMF.

It should be noted in conclusion that further detailed statistical investigation of the mean flows (for example, of the $\left\langle V_{y}\right\rangle$ IMF dependence) proved to be impossible. Such an analysis required formation of 16 data subsets, most of which included so few data points that the discovered differences between flows were statistically insignificant (see Sect. 2 for details). 

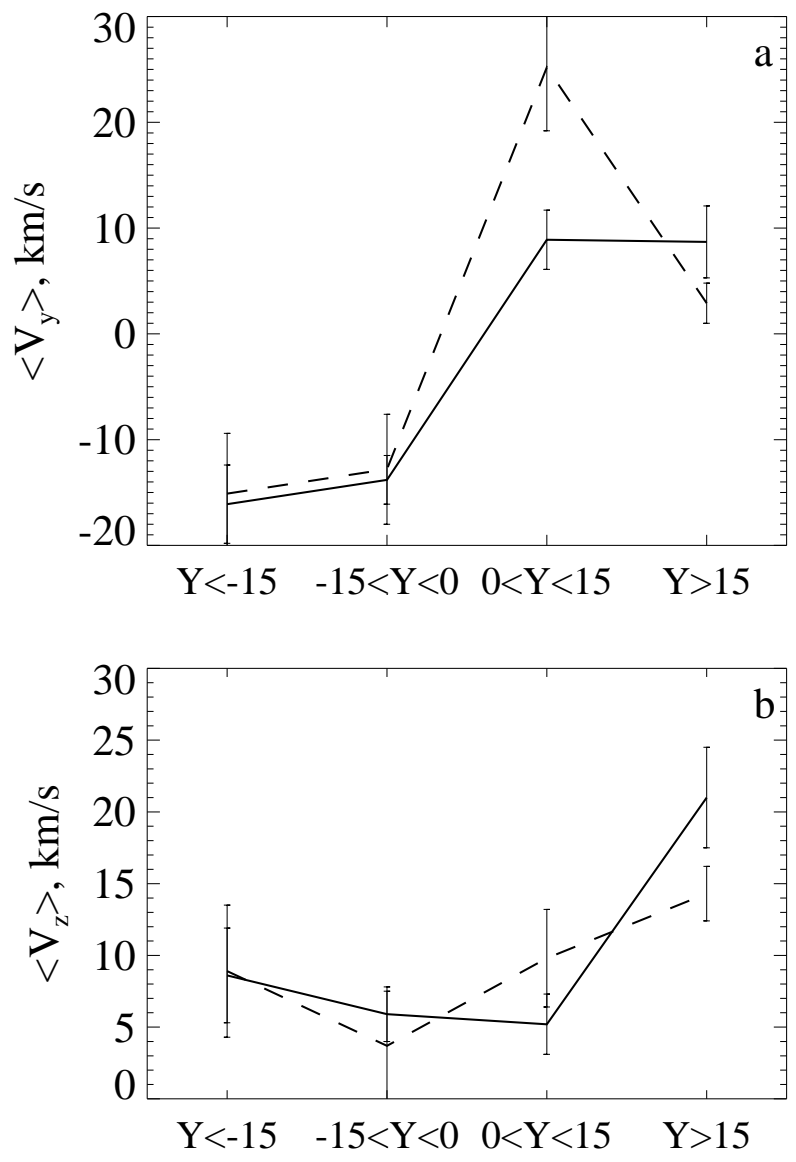

Fig. 5. Mean $V_{y}$ (a) and $V_{z}$ (b) velocities in the inner (dashed curves) and the outer (solid curves) plasma sheet with respect to the $Y$ GSM spacecraft position.

\section{Discussion and conclusions}

The design of the Interball-Tail spacecraft favors reliable measurements of plasma flows in the $Y Z$ GSM plane. An important issue in this context is the cross-check of the Interball statistical results on the $V_{y}$ velocity with the previous investigations of the AMPTE/IRM and ISEE-2 data. Average $V_{y}$ flows equal to $\sim 20-30 \mathrm{~km} / \mathrm{s}$ for $Y>0$ and equal to $\sim-15 \mathrm{~km} / \mathrm{s}$ for $Y<0$, agree both in direction and magnitude with the results of Angelopoulos et al. (1993, Fig 1). The $V_{y}$ PDFs measured by Interball-Tail are similar in their principal features to PDFs of ISEE-2 (Borovsky et al., 1997) and Geotail (Angelopoulos et al., 1999) measurements. All distributions are non-Gaussian, with significant high-velocity wings, and the mean velocities are much smaller than variances. Therefore, this part of our measurements is quite consistent with previous experiments.

The shape of the $V_{z}$ PDF proved to be close, in general, to the shape of the dawn-side $V_{y}$ PDF features, but the $V_{z}$ variance was smaller than the $V_{y}$ one, resulting in visually
Table 3. $\left\langle V_{z}\right\rangle$ statistics. All velocities are in $\mathrm{km} / \mathrm{s}$

\begin{tabular}{lccccc}
\hline IMF & $\left\langle V_{z}^{+}\right\rangle$ & $\left\langle V_{z}^{-}\right\rangle$ & $V_{d}$ & $V_{e}$ & $\delta V$ \\
\hline southward & 1.95 & 15.88 & 8.91 & 6.96 & 3.8 \\
northward & 3.57 & 2.68 & 3.12 & -0.44 & 2.1 \\
\hline
\end{tabular}

lower high-velocity wings. Both PDFs were symmetric after subtraction of the mean velocity values. On the contrary, the dusk-side $V_{y}$ PDF was skewed due to the excessive samples at positive high velocities. All distributions were readily fitted by the kappa function with exponent $k \sim 1-2$.

Though all PDFs were non-Gaussian, velocity variance is still a convenient simple measure of the scatter. The $V_{y}$ variations maximized in the pre-midnight part of the inner plasma sheet. The $V_{z}$ variations were always smaller than the $V_{y}$ ones, and maximized towards the dawn flank. One plausible source of ion velocity fluctuations is small-scale reconnection or BBF activity in the plasma sheet (Angelopoulos et al., 1992; Sergeev et al., 1996). Due to the magnetotail configuration, reconnection flow impulses should lay predominantly in the $X Y$ plane and occur in the vicinity of the field reversal region. Then, the observed maximum of the $V_{y}$ variance can be readily accounted for, since the substorm-related magnetotail activity is known to occur more often in the premidnight sector. However, properties of $V_{z}$ fluctuations cannot be explained by this mechanism.

Velocity variations can also be related to turbulent motions in the high-beta plasma (Borovsky et al., 1997). Some theories require the existence of such developed turbulence to explain the large-scale stability of the plasma sheet. Antonova and Ovchinnikov (1999) related the level of plasma turbulence to the thickness of the plasma sheet. In their model the dynamic balance of vertical turbulent diffusion and equatorward plasma convection determines the plasma sheet thickness. In the frame of this theory, the increase in the $V_{z}$ variance towards the dawn side indicates that the dawn plasma sheet is thicker if the large-scale convection is (at least statistically) uniform across the magnetotail.

Therefore, we can interpret the observed velocity variations as the combination of an inherent high-beta plasma turbulence, contributing more or less evenly to all components, and of a small-scale bursty activity, contributing mainly to $V_{y}$ (and $V_{x}$ ) components in the inner plasma sheet.

The large-scale kinetic model of ion dynamics in the magnetotail (Ashour-Abdalla et al., 1995) suggests that in addition to thermal ion population in the plasma sheet, there exists an accelerated plasma population in the form of smallsize beamlets. In such a case, flow variations are due to multiple crossings of these spatial structures. Our observations do not contradict this hypothesis. Since the beamlet model is essentially based on the computer simulations of the magnetotail, it should be possible to perform a direct comparison of observational results with the properties of numerical ion distributions. 
Detection of the average plasma sheet flows in the magnetotail is impeded by the high variance of velocity values. However, our data set was large enough to reveal small average vertical flows. In particular, the discovered equatorward convection can be compared with magnetospheric convection models (Nishida et al., 1998). Global convection schemes are essentially based on Dungey's model. They rely on penetration of the interplanetary motional electric field $E_{y}=V_{s w} \cdot B_{z}$ in the magnetosphere, when the magnetosphere is open via the dayside reconnection of magnetospheric and IMF field lines. Such reconnection is believed to occur when IMF $B_{z}<0$ and/or $\left|B_{z}\right|<\left|B_{y}\right|$. The magnitude of the resulting cross-tail electric field is estimated to be $10-15 \%$ of the original interplanetary $E_{y}$ : the size of the reconnection area at dayside is supposed to be $\sim 5 R_{E}$ (Burke et al., 1999) and the tail diameter is $\sim 30-40 R_{E}$. For our data set, the average negative IMF $B_{z}$ was $-3.0 \mathrm{nT}$, and the average solar wind velocity $V_{s w}$ was $444 \mathrm{~km} / \mathrm{s}$, so the predicted magnitude of the cross-tail convection field is 0.16 $0.22 \mathrm{mV} / \mathrm{m}$. When IMF is northward directed, the magnetosphere is closed for such penetration.

Experimentally found convection velocity is in agreement but approximately two times smaller than the model value. Equatorward velocity is associated with southward IMF $\left(B_{z}<0\right)$ was $\sim 7 \mathrm{~km} / \mathrm{s}$. Since the average local magnetic field $\left|B_{x}\right| \sim 13 \mathrm{nT}, 7 \mathrm{~km} / \mathrm{s}$ corresponds to the crosstail electric field $\sim 0.09 \mathrm{mV} / \mathrm{m}$. When IMF was northward $\left(B_{z}>0\right)$, this convection vanished.

Several factors can explain the difference between the measured convection and the model prediction for southward IMF. The model assumes that the IMF-related electric field penetrates all parts of the magnetosphere entrained in the convection process. However, while the existence of such an electric field in the magnetotail lobe is supported by the observations of the polar cap convection, the process of electric field penetration in the plasma sheet is less understood. It is likely less effective and requires favorable conditions. On the other hand, our statistical method is based on a singlepoint analysis, which is rather coarse and might not always perform optimal selections, partially mixing intervals with and without convection. Both of these factors should lead to an underestimation of the actual convection electric field.

Another interesting feature, revealed in the Interball data, is the northward plasma flow $\sim 8.9 \pm 3.8 \mathrm{~km} / \mathrm{s}$, which is also associated with southward IMF. Part of it is due to the relative motion of the plasma sheet and the spacecraft, amounting to $1 \mathrm{~km} / \mathrm{s}$ on average. The remaining $8 \pm 3.8 \mathrm{~km} / \mathrm{s}$ might be related to the BBF-type activity. During the winter seasons of the Northern Hemisphere, the plasma sheet is bent and shifted southward with respect to the Earth. Such quasi-stationary bending results in the existence of the effective northward component in earthward field-aligned plasma flows. In the modern neutral sheet models, the hinging point (behind which the sheet bending is believed to vanish) is placed at $X \sim-10 R_{E}$ (Fairfield, 1980), i.e. earthward from the region of our measurements. Nevertheless, the plasma sheet magnetic field lines behind the hinging point are shifted southward with respect to the Earth. When a field line is allowed to convect earthward (during bursts of the BBF-type activity), this shift might result in a small net northward flow.

The magnetotail flapping motion is also the plausible source of vertical plasma flows. Plasma bulk velocity during flaps can reach $50 \mathrm{~km} / \mathrm{s}$ and the characteristic period of tail oscillations is of the order of 1-20 min (Sergeev et al., 1998). Such velocity variations should, however, be averaged out in our data set. Indeed, each bin in the $V_{z}$ statistics includes about $4000 \mathrm{~min}$ of data, which represents about 200 periods of the presumably random flapping oscillations with the typical period of $20 \mathrm{~min}$.

In conclusion, the results of our investigation were quite consistent with the previous studies and, thanks to the design of the instrument, provided the first experimental evidence of vertical convection in the plasma sheet. The discovered asymmetry of velocity fluctuations should be taken into account during modern theoretical analysis and simulations.

Acknowledgement. The authors would like to thank A. O. Fedorov for conducting the CORALL experiment, S. I. Klimov and S. A. Romanov for conducting the Interball-Tail magnetic field experiment, L. M. Zelenyi and E. E. Antonova for useful discussions. We are grateful to R. P. Lepping and K. Ogilvie for available Wind data and to NASA GSFC for maintaining the public CDAWeb database. The work was supported by the INTAS Grant 97-1612.

Topical Editor G. Chanteur thanks E. E. Antonova and another referee for their help in evaluating this paper.

\section{References}

Angelopoulos, V., Baumjohann, W., Kennel, C. F., Coroniti, F. V., Kivelson, M. G., Pellat, R., Walker, R. J., Lühr, H., and Paschmann, G.: Bursty bulk flows in the inner central plasma sheet, J. Geophys. Res., 97, 4027-4039, 1992.

Angelopoulos, V., Kennel, C. F., Coroniti, F. V., Pellat, R., Spence, H. E., Kivelson, M. G., Walker, R. J., Baumjohann, W., Feldman, W. C., Gosling, J. T., and Russell, C. T.: Characteristics of ion flow in the quiet state of the inner plasma sheet, Geophys. Res. Lett., 20, 1711-1714, 1993.

Angelopoulos, V., Kennel, C. F., Coroniti, F. V., Pellat, R., Kivelson, M. G., Walker, R. J., Russell, C. T., Baumjohann, W., Feldman, W. C., and Gosling, J. T.: Statistical characteristics of bursty bulk flow events, J. Geophys. Res., 99, 21 257-21 280, 1994.

Angelopoulos, V., Mukai, T., and Kokubun, S.: Evidence for intermittency in Earth's plasma sheet and implications for selforganized criticality, Phys. of Plasmas, 6, 4161-4168, 1999.

Antonova, E. E. and Ovchinnikov, I. L.: Magnetostatically equilibrated plasma sheet with developed medium-scale turbulence: Structure and implications for substorm dynamics, J. Geophys. Res., 104, 17 289-17 298, 1999.

Ashour-Abdalla, M., Zelenyi, L. M., Peroomian, V., Richard, R. L., and Bosqued, J. M.: The mosaic structure of plasma bulk flows in the Earth's magnetotail, J. Geophys. Res., 100, 19 191-19210, 1995.

Bame, S. J., Asbridge, J. R., Felthauser, H. E., Glore, J. P., Paschmann, G., Hemmerich, P., Lehmann, K., and Rosenbauer, H.: ISEE-1 and ISEE-2 fast plasma experiment and the 
ISEE-1 solar wind experiment, IEEE Trans. Geosci. Electr., 16, 216-220, 1978.

Baumjohann, W., Paschmann, G., and Lühr, H.: Characteristics of high-speed ion flows in the plasma sheet, J. Geophys. Res., 95, 3801-3809, 1990.

Borovsky, J. E., Elphic, R. C., Funsten, H. O., and Thomsen, M. F.: The Earth's plasma sheet as a laboratory for flow turbulence in high $\beta$ MHD, J. Plasma Phys., 57, 1-34, 1997.

Burke, W. J., Weimer, D. R., and Maynard, N. C.: Geoeffective interplanetary scale sizes derived from regression analysis of polar cap potentials, J. Geophys. Res., 104, 9989-9994, 1999.

Dungey, J. W.: Interplanetary magnetic field and the auroral zones, Phys. Rev. Lett., 6, 47-48, 1961.

Fairfield, D. H.: A statistical determination of the shape and position of the geomagnetic neutral sheet, J. Geophys. Res., 85, 775-780, 1980.

Klimov, S., Romanov, S., Amata, E., Blecki, J., Büchner, J., Juchniewicz, J., Rustenbach, J., Triska, P., Woolliscroft, L. J. C., Savin, S., Afanas'yev, Y., de Angelis, U., Auster, U., Bellucci, G., Best, A., Farnik, F., Formisano, V., Gough, P., Grard, R., Grushin, V., Haerendel, G., Ivchenko, V., Korepanov, V., Lehmann, H., Nikutowski, B., Nozdrachev, M., Orsini, S., Parrot, M., Petrukovich, A., Rauch, J. L., Sauer, K., Skalsky, A., Slominski, J., Trotignon, J. G., Vojta, J., and Wronowski, R.: ASPI experiment: measurements of fields and waves onboard the INTERBALL-1 spacecraft, Ann. Geophysicae, 15, 514-527, 1997.

Nishida, A., Mukai, T., Yamamoto, T., Kokubun, S., and Maezawa, K.: A unified model of the magnetotail convection in geomagnetically quiet and active times, J. Geophys. Res., 103,
4409-4418, 1998

Paschmann, G., Loidl, H., Obermayer, P., Ertl, M., Laborenz, R., Sckopke, N., Baumjohann, W., Carlson, C. W., and Curtis, D. W.: The plasma instrument for AMPTE/IRM, IEEE Trans. Geosci. Rem. Sens., 23, 262-266, 1985.

Pontius, Jr., D. H. and Wolf, R. A.: Transient flux tubes in the terrestrial magnetosphere, Geophys. Res. Lett., 17, 49-52, 1990.

Sergeev, V. A. and Lennartsson, W.: Plasma sheet at $X \approx 20 R_{E}$ during steady magnetospheric convection, Planet. Space Sci., 36, 353-370, 1988.

Sergeev, V. A., Pulkkinen, T. I., and Pellinen, R. J.: Coupled-mode scenario for the magnetospheric dynamics, J. Geophys. Res., 101, 13 047-13 065, 1996.

Sergeev, V. A., Angelopoulos, V., Carlson, C., and Sutcliffe, P.: Current sheet measurements within a flapping plasma sheet, J. Geophys. Res., 103, 9177-9187, 1998.

Vasyliunas, V. M.: A survey of low energy electrons in the evening sector of the magnetosphere with OGO-1 and OGO-3, J. Geophys. Res., 73, 2839-2884, 1968.

Yermolaev, Y. I., Fedorov, A. O., Vaisberg, O. L., Balebanov, V. M., Obod, Y. A., Jimenez, R., Fleites, J., Llera, L., and Omelchenko, A. N.: Ion distribution dynamics near the Earth's bow shock: first measurements with the 2D ion energy spectrometer CORALL on the INTERBALL/Tail-probe satellite, Ann. Geophysicae, 15, 533-541, 1997.

Yermolaev Y. I., Petrukovich, A. A., Zelenyi, L. M., Antonova, E. E., Ovchinnikov, I. L., and Sergeev, V. A.: Investigation of the structure and dynamics of plasma sheet: The CORALL experiment of the Interball project, Cosmic Research, 38, 13-19, 2000. 